\title{
Evaluation of the Effectiveness of Cervical Cancer Screening: A Case-Control Study in Miyagi, Japan
}

\author{
Hiromitsu Makino, Shinji Sato, Akira Yajima, Shoko \\ Komatsu* and Akira Fukao* \\ Department of Obstetrics and Gynecology, and * Department \\ of Public Health, Tohoku University School of Medicine, \\ Sendai 980-7ry
}

\begin{abstract}
Makino, H., Sato, S., Yajima, A., Komatsu, S. and Fukao, A. Evaluation of the Effectiveness of Cervical Cancer Screening: A Case-Control Study in Miyagi, Japan. Tohoku J. Exp. Med., 1995, 175 (3), 171-178— To estimate the effectiveness of screening for invasive cervical cancer, a case-control study was performed in Miyagi, Japan. A total of 198 cases (129 mass screen-detected and 69 outpatient-detected) of invasive cervical cancer were identified between 1984 and 1990. The results of the Papanicolaou smear of these cases were compared to those of 396 age- ( \pm 5 years) and area-matched controls. Compared with women who had no prior screening (cases $51.6 \%$, controls $16.2 \%$ ), women who were screened had an odds ratio (OR) for invasive cervical cancer of 0.14 (95\% confidence interval [CI] 0.088-0.230). The $\mathrm{OR}$ for the 175 cases of squamous cell carcinoma was 0.13 (95\% CI $0.077-0.215)$, while the OR for the 23 cases of screened women with adenocarcinoma was 0.40 (95\% CI 0.091-1.753). The time intervals following the last negative smear were assessed and we found an OR for a one-year interval and a two-year interval of 0.09 (95\% CI $0.055-0.163)$ and $0.17(95 \% \mathrm{CI}$ 0.083-0.335), respectively. In conclusion, screening for invasive cervical cancer is not only effective but also the degree of protection is high for one to two years. cervical cancer; case-control study; cervical cancer screening
\end{abstract}

Miyagi is one of the first areas in Japan to be involved in mass screening for cervical cancer. From 1984-1990, in Miyagi, a total of one million Papanicolaou (Pap) smears were taken among women over the age of 30 . The cytodiagnoses of the Pap smears for these women were performed primarily at the Center for Clinical Cytology (Miyagi Cancer Society). The results of the screening program and the relationship between histological features and history of prior cervical screening have previously been reported by our group (Yajima et al. 1979; 1982). However, the effectiveness of this form of screening for cervical cancer in Miyagi using epidemiological methods has not been described before except for a prelimi-

Received September 20, 1994; revision accepted for publication November 29, 1994.

Address for reprints: Hiromitsu Makino, Department of Obstetrics and Gynecology, Tohoku University School of Medicine, 1-1 Seiryo-machi, Aoba-ku, Sendai 980-77, Japan. 
nary analysis (Makino et al. 1991).

The best method for evaluating the effectiveness of screening is a randomized controlled trial. However, because mass screening for cervical cancer is now widespread and because it has become difficult to obtain a control group, this method is no longer practical. As an alternative, case-control studies have been proposed (Miller 1985; Sasco et al. 1986). The first case-control study of cervical screening was performed and reported by Clarke and Anderson (1979). Subsequently several other studies (Aristizabal et al. 1984; Macgregor et al. 1985; Clarke et al. 1986; van der Graaf et al. 1988; Olesen 1988; Celentano et al. 1988; Palli et al. 1990) were performed in other centers. Although a case-control study of cervical cancer screening has been conducted in Osaka, Japan, the odds ratios (ORs) for the "ever" vs. the "never" screened group in that study were not significantly different. This was not because the screening effect was small, but because of the small number of cases (Sobue et al. 1988).

The purpose of the present study was to evaluate the effectiveness of screening for invasive cervical cancer in Japan by means of a case-control study utilizing a large number of cases.

\section{Subjects ANd Methods}

The study population consisted of 198 cases of documented invasive cervical cancer occurring within the Miyagi Prefecture between April 1, 1984, and March 31, 1990. One hundred twenty-nine of these cases were detected by mass screening for cervical cancer (mass screen-detected cases). The remaining 69 cases were outpatients with gynecological symptoms (i.e.: genital bleeding, discharge, or pelvic pain) and had documented cervical smears (outpatient-detected cases). These patients ranged in age from 35-79 years and had a histological diagnosis of invasive cervical cancer established within six months of the detection of an abnormal smear (Pap class III V) at the Center for Clinical Cytology.

This Center is not only responsible for all cytological and histological diagnoses obtained during mass screening but also examines approximately $90 \%$ of the cervical smears obtained from outpatients who visit gynecologists within the Miyagi Prefecture. The cytological results of both screened patients and outpatients are maintained in the cytology file of the Center. Thus, both cases and controls can be identified by this cytology file. Two controls were matched for each case by age ( \pm 5 years) and district of residence. Women without invasive cervical cancer served as controls. Their record numbers were the nearest to the cases on the cytology file. The controls for the mass screen-detected cases were selected from women who had participated in the mass screening program, while controls for the outpatient-detected cases were selected from women who had visited gynecologists and had cervical smear examinations.

We investigated the past screening history of both cases and controls using past cytology files and self-completed questionnaires. If the screening history on 
the cytology file was inconsistent with data obtained from the questionnaire, the screening history from the questionnaire was used. The reason is that the cytodiagnosis was occasionally performed in institutes other than this Center. The screening performed at the time of diagnosis was not included in the past screening history of cases or controls. Furthermore, women who had a history of a prior hysterectomy or previous abnormal cytology were excluded from the study.

The OR for the occurrence of invasive cervical cancer in women who had been previously screened with one or more Pap smears compared to women who had never been screened was calculated. In addition, we estimated the OR for the occurrence of invasive cervical cancer during each year after the last negative screening. A conditional logistic regression analysis was performed using the SAS statistical package to estimate the OR and determine statistical significance (Breslow and Day 1980).

\section{Results}

Table 1 demonstrates the age distribution of cases in our study. In mass screen-detected cases, $47.2 \%$ were women between the ages of 35 and 44, with a mean age of 48.9 years. Among outpatient-detected cases, the percent distribution by 10-year age brackets was similar between 35 and 74 years of age, with an average age of 54.8 years. Squamous cell carcinoma accounted for $88.4 \%$ of these cases while adenocarcinoma accounted for $11.6 \%$ of the total cases. The mean age was 51.1 years for patients with squamous cell carcinoma and 49.7 years for patients with adenocarcinoma.

Table 2 shows the OR for women who had undergone screening compared to women who had never been screened. The OR was 0.14 for both the mass screening group (95\% CI 0.077-0.263) and the outpatient group (95\% CI 0.066$0.309)$. The OR was $0.14(95 \%$ CI $0.088-0.230)$ for all groups.

The ORs according to histological type and age are estimated in Table 3.

TABLE 1. Distribution of case by age

\begin{tabular}{cccc}
\hline $\begin{array}{c}\text { Age } \\
\text { (years) }\end{array}$ & $\begin{array}{c}\text { Mass screen- } \\
\text { detected cases }\end{array}$ & $\begin{array}{c}\text { Outpatient- } \\
\text { detected cases }\end{array}$ & Total cases \\
\hline $35-44$ & $61(47.2)$ & $20(29.0)$ & $81(41.0)$ \\
$45-54$ & $29(22.5)$ & $14(20.3)$ & $43(21.7)$ \\
$55-64$ & $24(18.6)$ & $16(23.2)$ & $40(20.2)$ \\
$65-74$ & $13(10.1)$ & $14(20.3)$ & $27(13.6)$ \\
$75-79$ & $2(1.6)$ & $5(7.2)$ & $7(3.5)$ \\
Total & $129(100)$ & $69(100)$ & $198(100)$ \\
Mean age & 48.9 & 54.8 & 50.9 \\
\hline ( ): \% & &
\end{tabular}


TABLE 2. Odds ratios: "ever" vs. "never" screened

\begin{tabular}{lccrlc}
\hline & $\begin{array}{c}\text { Screening } \\
\text { history }\end{array}$ & Cases & Controls & $\begin{array}{l}\text { Odds } \\
\text { ratio }\end{array}$ & $\begin{array}{c}95 \% \text { confidence } \\
\text { interval }\end{array}$ \\
\hline Mass screening group & Ever & $74(57.4)$ & $231(89.5)$ & $0.14^{* * *}$ & $0.077-0.263$ \\
& Never & $55(42.6)$ & $27(10.5)$ & 1.0 & \\
Outpatient group & Ever & $23(33.3)$ & $101(73.2)$ & $0.14^{* * *}$ & $0.066-0.309$ \\
& Never & $46(66.7)$ & $37(26.8)$ & 1.0 & \\
Total group & Ever & $97(49.0)$ & $332(83.8)$ & $0.14^{* * *}$ & $0.088-0.230$ \\
& Never & $101(51.0)$ & $64(16.2)$ & 1.0 & \\
\hline
\end{tabular}

$$
\begin{aligned}
& { }^{* * *} p<0.001 \\
& \text { ( }): \%
\end{aligned}
$$

TABLE 3. Odds ratios according to histological type and age

\begin{tabular}{cccccc}
\hline & $\begin{array}{c}\text { Screening } \\
\text { history }\end{array}$ & Cases & Controls & $\begin{array}{c}\text { Odds } \\
\text { ratio }\end{array}$ & $\begin{array}{c}95 \% \text { confidence } \\
\text { interval }\end{array}$ \\
\hline $\begin{array}{c}\text { Histological type } \\
\text { Squamous cell- } \\
\text { carcinoma }\end{array}$ & Ever & $80(45.7)$ & $293(83.7)$ & $0.13^{* * *}$ & $0.077-0.215$ \\
Adenocarcinoma & Never & $95(54.3)$ & $57(16.3)$ & 1.0 & \\
& Ever & $17(73.9)$ & $39(84.8)$ & 0.40 n.s. & $0.091-1.753$ \\
Age (years) & Never & $6(26.1)$ & $7(15.2)$ & 1.0 & \\
$35-49$ & & & & & \\
& Ever & $52(50.0)$ & $180(86.5)$ & $0.15^{* * *}$ & $0.078-0.280$ \\
$50-79$ & Never & $52(50.0)$ & $28(13.5)$ & 1.0 & \\
& Ever & $45(47.9)$ & $153(81.4)$ & $0.14^{* * *}$ & $0.065-0.281$ \\
& Never & $49(52.1)$ & $35(18.6)$ & 1.0 & \\
\hline
\end{tabular}

$$
\begin{aligned}
& { }^{* * *} p<0.001 \\
& \text { n.s., not significant. } \\
& \text { ( }): \%
\end{aligned}
$$

TABLE 4. Odds ratios: interval between the last negative screening result and diagnosis

\begin{tabular}{lcccccc}
\hline \multicolumn{1}{c}{$\begin{array}{c}\text { Interval } \\
\text { (years) }\end{array}$} & 1 & 2 & 3 & 4 & $\geqq 5$ & Total \\
\hline Cases & $43(21.7)$ & $18(9.1)$ & $10(5.1)$ & $6(3.0)$ & $121(61.1)$ & $198(100)$ \\
Controls & $231(58.3)$ & $56(14.1)$ & $11(2.8)$ & $7(1.8)$ & $91(23.0)$ & $396(100)$ \\
Odds ratio & $0.09^{* * *}$ & $0.17^{* * *}$ & 0.67 & 0.45 & 1.0 & \\
$95 \%$ confidence interval & $0.055-$ & $0.083-$ & $0.259-$ & $0.125-$ & & \\
& 0.163 & 0.335 & 1.727 & 1.593 & & \\
\hline
\end{tabular}

$$
{ }^{* * *} p<0.001
$$$$
\text { ( ): } \%
$$ 
For the 175 cases of squamous cell carcinoma, the OR of screened women was 0.13 (95\% CI $0.077-0.215$ ), while the OR was 0.40 for the 23 cases of adenocarcinoma (95\% CI 0.091-1.753). For the 35-49 year age group, screened women had an OR of 0.15 (95\% CI 0.078-0.280). For the 50-79 year age group, the OR was $0.14(95 \%$ CI $0.065-0.281)$.

Table 4 shows the ORs for the interval of years between the last negative screening result and diagnosis. We observed an OR of 0.09 (95\% CI 0.055-0.163) for the 1 year period after the last negative smear and 0.17 (95\% CI 0.083-0.335) for the 2 year period after the last negative smear. The ORs at three and four years were 0.67 (95\% CI 0.259-1.727) and 0.45 (95\% CI 0.125-1.593), respectively.

\section{Discussion}

The introduction of bias may complicate case-control studies (Sackett 1979). In population-based studies, self-selection bias usually cannot be avoided and tends to overestimate the benefit of mass screening. In our study, however, self-selection bias was minimized by using controls selected from women who participated in mass screening (in mass screen-detected cases) and from women who had gynecological symptoms (in outpatient-detected cases). Because the cytological examinations were occasionally performed in other institutes, past screening histories were investigated using self-completed questionnaires in additon to cytology files of this Center. Therefore, recall bias cannot be completely excluded. Although in mass screen-detected cases there is a tendency to detect asymptomatic cancers more frequently (length bias), our cases involved not only mass screen-detected cases but also outpatient-detected cases, some of which had rapidly growing and advanced tumors.

In recent case-control studies (Aristizabal et al. 1984; van der Graaf et al. 1988; Palli et al. 1990), the ORs for "ever" vs. "never" screened subjects have ranged from 0.10 to 0.32 . Our data are consistent with these reports (OR 0.14). Additionally, we estimated the ORs by two age groups (35-49 years vs. 50-79 years) and found no difference between the ORs of these two groups. Moss (1986) and Celentano et al. (1988) have obtained similar results. Thus there is no evidence to implicate age-related effects in any of these three studies. Further studies are required to arrive at a conclusive opinion.

Our study also examined the time interval from the last negative Pap smears. The OR since the last negative smear was 0.09 for the 1-year interval and 0.17 for 2-year interval. The ORs were statistically significant for both time periods. Clarke et al. (1986) have observed an OR of 0.21 for invasive cervical cancer for intervals of less than 12 months after a negative smear and 0.28 for intervals between 12 to 23 months. Macgregor et al. (1985) have demonstrated ORs of 0.11 for intervals between 12 to 23 months and 0.29 for intervals between 24 to 35 months. Finally Olesen (1988) and Celentano et al. (1988) have estimated ORs of 0.15 for women screened every three years and 0.29 for intervals of less than 
three years.

In Japan, annual screening for cervical cancer is currently recommended by public health officials. However the number of voluntary participants in the screening program still has not appreciably increased and the participants in the program tend to be the same every time. Most participants in the program had Pap smears performed either annually or every other year. There were only a few participants whose screening intervals were longer than 3 years. Thus, it is difficult to evaluate results beyond this 3-year period.

There are two histological forms of cervical cancer. Although the incidence rate of adenocarcinoma is increasing (Davis and Moon 1975; Shingleton et al. 1981), the natural history and cytodiagnostic criteria for establishing the diagnosis of adenocarcinoma remain unclear. Our study is consistent with the study performed by Clarke and Anderson (1979) (OR 0.7) that suggested that Pap smears might be less effective when screening for adenocarcinoma as opposed to screening for squamous cell carcinoma although the number of cases of adenocarcinoma in our and their studies is small. Moreover, the false negative cytology rates for adenocarcinoma are higher than those for squamous cell carcinoma (Morell et al. 1982; Makino et al. 1993). These issues need to be considered when attempting to improve quality control for Pap smear screening.

The incidence of cervical cancer is closely linked to reproductive behavior. Factors associated with the disease include age at first intercourse, number of sexual partners, age at marriage, number of pregnancies and children, and socioeconomic status (Martin 1967; Cuzick et al. 1989). Due to insufficient information, however, we were unable to adjust for these confounding factors in this study.

In conclusion, using a case-control approach, our study evaluated the effectiveness of screening for invasive cervical cancer in Japan. Further studies are currently being designed to adjust for various other factors known to be associated with the disease.

\section{Acknowledgments}

We wish to thank the physicians who cooperated in this study as well as the staff of the Miyagi Cancer Society. This work was supported in part by a Grant-in-Aid from the Ministry of Health and Welfare for the Comprehensive 10-Year Strategy for Cancer Control and in part by a Grant-in-Aid for Scientific Research from the Ministry of Education, Science and Culture, Japan.

\section{References}

1) Aristizabal, N., Cuello, C., Correa, P., Collazos, T. \& Haenszel, W. (1984) The impact of vaginal cytology on cervical cancer risks in Cali, Colombia. Int. J. Cancer, 34, 59.

2) Breslow, N.E. \& Day, N.E. (1980) Statistical Methods in Cancer Research, vol. 1 The analysis of case-control studies. IARC Scientific Publications, No. 32, International 
Agency for Research on Cancer, Lyon.

3) Celentano, D.D., Klassen, A.C., Weisman, C.S. \& Rosenshein, N.B. (1988) Cervical cancer screening practices among older women: Results from the Maryland cervical cancer case-control study. J. Clin. Epidemiol., 41, 531-541.

4) Clarke, E.A. \& Anderson, T.W. (1979) Does screening by "Pap" smears help prevent cervical cancer? A case-control study. Lancet, ii, 1-4.

5) Clarke, E.A., Hilditch, S. \& Anderson, T.W. (1986) Optimal frequency of screening for cervical cancer: A Toronto case-control study. In: Screening for Cancer of the Uterine Cervix, edited by M. Hakama, A.B. Miller \& N.E. Day, IARC Scientific Publications, No. 76, International Agency for Research on Cancer, Lyon, pp. 125-131.

6) Cuzick, J., De Stavola, B., McCance, D., Ho, T.H., Tan, G., Cheng, H., Chew, S.Y. \& Salmon, Y.M. (1989) A case-control study of cervix cancer in Singapore. Br. J. Cancer, 60, 238-243.

7) Davis, J.R. \& Moon, L.B. (1975) Increased incidence of adenocarcinoma of uterine cervix. Obstet. Gynecol., 45, 79-83.

8) Macgregor, J.E., Moss, S.M., Parkin, D.M. \& Day, N.E. (1985) A case-control study of cervical cancer screening in north east Scotland. Br. Med. J., 290, 1543-1546.

9) Makino, H., Sato, S., Yajima, A. \& Fukao, A. (1991) Case-control study of the effectiveness of mass screening in reducing invasive cervical cancer. Acta Obstet. Gynecol. Jpn., 43, 1226-1232. (in Japanese)

10) Makino, H., Nakanomyo, Y., Sato, S., Yajima, A. \& Higashiiwai, H. (1993) False negative cytology in mass screening for cervical cancer. J. Jpn. Soc. Clin. Cytol., 32, 906-913. (in Japanese)

11) Martin, C.E. (1967) Marital and coital factors in cervical cancer. Am. J. Pub. Health, 57, 803-814.

12) Miller, A.B. (1985) Principles of screening and of the evaluation of screening programs. In: Screening for Cancer, edited by A.B. Miller, Academic Press, Inc., Orlando, pp. 3-24.

13) Morell, N.D., Taylor, J.R., Snyder, R.N., Ziel, H.K., Saltz, A. \& Willie, S. (1982) False-negative cytology rates in patients in whom invasive cervical cancer subsequently developed. Obstet. Gynecol., 60, 41-45.

14) Moss, S. (1986) Combined analysis of data from north-east Scotland and Iceland. In: Screening for Cancer of the Uterine Cervix, edited by M. Hakama, A.B. Miller \& N.E. Day, IARC Scientific Publications, No. 76, International Agency for Research on Cancer, Lyon, pp. 43-46.

15) Olesen, F. (1988) A case-control study of cervical cytology before diagnosis of cervical cancer in Denmark. Int. J. Epidemiol., 17, 501-508.

16) Palli, D., Carli, S., Cecchini, S., Venturini, A., Piazzesi, G. \& Buiatti, E. (1990) A centralised cytology screening programme for cervical cancer in Florence. $J$. Epidemiol. Community Health, 44, 47-51.

17) Sackett, D.L. (1979) Bias in analytic research. J. Chron. Dis., 32, 51-63.

18) Sasco, A.J, Day, N.E. \& Walter, S.D. (1986) Case-control studies for the evaluation of screening. J. Chron. Dis., 39, 399-405.

19) Shingleton, H.M., Gore, H., Bradley, D.H. \& Soong, S.-J. (1981) Adenocarcinoma of the cervix. 1. Clinical evaluation and pathologic features. Am. J. Obstet. Gynecol., 139, 799-814.

20) Sobue, T., Suzuki, T., Hashimoto, S., Yokoi, N. \& Fujimoto, I. (1988) A case-control study of the effectiveness of cervical cancer screening in Osaka, Japan. Jpn. J. Cancer Res. (Gann), 79, 1269-1275.

21) van der Graaf, Y., Zielhuis, G.A., Peer, P.G.M. \& Vooijs, P.G. (1988) The effectiveness of cervical screening: A population-based case-control study. J. Clin. Epidemiol., 41, 21-26.

22) Yajima, A., Higashiiwai, H., Sato, A., Watanabe, M., Hoshi, K., Mori, T., Yonemoto, 
Y., Suzuki, M. \& Noda, K. (1979) Mass population screening for cervical cancer in Miyagi prefecture. Gynecol. Oncol., 8, 131-140.

23) Yajima, A., Mori, T., Sato, S., Wakisaka, T. \& Suzuki, M. (1982) Effect of cytologic screening on the detection of cervical carcinoma. Obstet. Gynecol., 59, 565-568. 